\title{
Long-Term Ketogenic Diet Induces Metabolic Acidosis, Anemia, and Oxidative Stress in Healthy Wistar Rats
}

\author{
Aryadi Arsyad (i), ${ }^{1}$ Irfan Idris, ${ }^{1}$ Andi A. Rasyid, ${ }^{2}$ Rezky A. Usman, ${ }^{2}$ Kiki R. Faradillah, \\ Wa Ode U. Latif, ${ }^{2}$ Zidni I. Lubis, ${ }^{3}$ Aminuddin Aminuddin, ${ }^{4}$ Ika Yustisia, ${ }^{5}$ \\ and Yulia Y. Djabir ${ }^{6}$ \\ ${ }^{1}$ Department of Physiology, Faculty of Medicine, Hasanuddin University, Makassar, Indonesia \\ ${ }^{2}$ Biomedical Science Study Program, Postgraduate School, Hasanuddin University, Makassar, Indonesia \\ ${ }^{3}$ Department of Physiotherapy, Faculty of Health Science, University of Muhammadiyah Malang, Malang, Indonesia \\ ${ }^{4}$ Department of Nutrition, Faculty of Medicine, Hasanuddin University, Makassar, Indonesia \\ ${ }^{5}$ Department of Biochemistry, Faculty of Medicine, Hasanuddin University, Makassar, Indonesia \\ ${ }^{6}$ Laboratory of Clinical Pharmacy, Faculty of Pharmacy, Hasanuddin University, Makassar, Indonesia \\ Correspondence should be addressed to Aryadi Arsyad; aryadi.arsyad@gmail.com
}

Received 13 December 2019; Revised 19 April 2020; Accepted 21 May 2020; Published 29 June 2020

Academic Editor: Phillip B. Hylemon

Copyright ( 2020 Aryadi Arsyad et al. This is an open access article distributed under the Creative Commons Attribution License, which permits unrestricted use, distribution, and reproduction in any medium, provided the original work is properly cited.

\begin{abstract}
Background. Ketogenic diet has been used as supportive therapy in a range of conditions including epilepsy, diabetes mellitus, and cancer. Objective. This study aimed to investigate the effects of long-term consumption of ketogenic diet on blood gas, hematological profiles, organ functions, and superoxide dismutase level in a rat model. Materials and Methods. Fifteen male Wistar rats were divided into control $(n=8)$ and ketogenic $(n=7)$ groups. Controls received standard diet contained $52.20 \%$ of carbohydrates, $7.00 \%$ fat, and $15.25 \%$ protein; meanwhile, the ketogenic group received a high-fat-low-carbohydrate diet which contained $5.66 \%$ of carbohydrate, $86.19 \%$ fat, and $8.15 \%$ protein. All rats were caged individually and received $30 \mathrm{~g}$ of either standard or high-fat-low-carbohydrate pellets. The experiment was carried out for 60 days before the blood samples were taken and analyzed to obtain blood gas, cell counts, organ biomarkers, and plasma antioxidant superoxide dismutase (SOD) levels. Results. The rats subjected to ketogenic diet experienced a marked decrease in body weight, blood sugar, and increased blood ketones $(p<0.05)$. The average blood $\mathrm{pH}$ was $7.36 \pm 0.02$ and base excess was $-5.57 \pm 2.39 \mathrm{mOsm} / \mathrm{L}$, which were significantly lower than controls $(p<0.05)$. Hematological analysis showed significantly lower erythrocyte, hemoglobin, and hematocrit levels. No significant changes were found in alanine aminotransferase, aspartate aminotransferase, urea, and creatinine levels, indicating normal liver and kidney functions. Nevertheless, plasma SOD level significantly reduced with ketogenic diet. Conclusion. Longterm ketogenic diet induces metabolic acidosis, anemia, and reduced antioxidant enzyme level in rats following 60 days of consuming high-fat-low-carbohydrate diet.
\end{abstract}

\section{Introduction}

The ketogenic diet is a food regimen which consists of a high concentration of fat, with moderate/low protein and very low carbohydrate content. This type of diet triggers high production of ketone bodies derived from the breakdown of fat to produce energy [1]. Some studies show that the ketogenic diet has therapeutic benefits in a range of illnesses. It has been recommended as a supplementary therapy for polycystic ovary syndrome, acne, cancer, and respiratory distress [2]. It is also beneficial as anticonvulsant therapy to reduce the frequency of seizures in people with epilepsy $[3,4]$. Ketogenic diet may also help to reduce HbA1C levels in people with type 2 diabetes, maintaining mood stability for people with bipolar disorder, and reducing cholesterol levels in obese patients [5].

A clinical study has demonstrated that a short-term ketogenic diet for 14 days might increase the concentration 
of ketone bodies in the blood, but it also improved the antioxidant capacity of the blood that contributes to reduced oxidative stress [6]. Another clinical trial has shown that consuming ketogenic diet for 20 days significantly reduced carbon dioxide deposits in the body, which may find clinical benefit in patients with increased $\mathrm{PaCO}_{2}$ due to respiratory failure [2].

Despite its popular use, some concerns arise on how ketogenic diet will affect the whole-body system. Since ketogenic diet replaces glucose with fat as the main source of energy, the body is forced to activate a series of fat metabolic processes to acquire energy [7]. Fat metabolic processes form acetyl coenzyme A (acetyl-CoA) as the main product, which then enters the citric acid cycle and is oxidized to produce ATP [8]. Acetyl-CoA that exceeds the availability of oxaloacetate and/or the activity of the citric acid cycle leads to an increase in ketone bodies (acetoacetate, $\beta$-hydroxybutyrate, and acetone). This process is called ketogenesis [9]. The ketone bodies formed from ketogenic diets are acidic; therefore, excessive excretion of these acids through kidneys may cause a decrease in alkaline reserves or bicarbonate ions $\left(\mathrm{HCO}_{3}{ }^{-}\right)$[10]. As a result, the implication of ketogenic diet reduced blood $\mathrm{pH}$, leading to ketoacidosis [11].

Several animal models have been used to learn about the effect of a high-fat diet on the function of vital organs, such as the kidneys and liver [12,13]. High-fat diet is more likely to trigger a reduction in mitochondrial quinone pool and is associated with increased mitochondrial reactive oxygen species (ROS) formation in the rat liver [14]. A high-fat diet has been shown to induce alteration in renal lipid metabolism in mice, especially the balance between lipogenesis and lipolysis, leading to the accumulation of lipid in the kidneys and, consequently, renal dysfunction [15].

To obtain more comprehensive data on how ketogenic diet may affect the whole-body system, this present study aimed to investigate the effects of long-term consumption of ketogenic diet on blood gas profiles, hematological parameters, organ functions, and antioxidant level in a rat model.

\section{Materials and Methods}

2.1. Preparation of Standard and Ketogenic Diet. Standard food was obtained from a manufacturer as standard pellets for rodents $\left(\mathrm{AD}{ }^{\circledR}{ }^{\circledR}\right.$, Indonesia), while the ketogenic food was prepared in our laboratory by involving a nutritionist. The ketogenic pellets contain 30\% of nonpure fat mixed with $70 \%$ of goat fat (Table 1 ), which is formulated based on NutriSurvey ${ }^{\circledR}$ software to calculate the calorie intake and the percentage of macro and micronutrients per gram pellet. All ingredients were liquefied and mixed using a hand mixer and then frozen for 24 hours with the temperature of $-20^{\circ} \mathrm{C}$. The solidified material was then pulverized and molded into pellets. The standard and ketogenic pellets were then examined for their fat, protein, and carbohydrate contents at the Laboratory of Animal Food Chemistry, Faculty of Animal Science, Universitas Hasanuddin.
Table 1: Composition of standard and ketogenic diet.

\begin{tabular}{lc}
\hline Composition & Percentage \\
\hline Standard diet ${ }^{\mathrm{a}}$ & \\
Water & 12 \\
Protein & 15 \\
Palm oil & 7 \\
Fiber & 6 \\
Calcium & 7 \\
Phosphor & 0.7 \\
Enzyme & 0.1 \\
Corns & 52.2 \\
\hline Ketogenic diet ${ }^{\mathrm{b}}$ & \\
Water & - \\
Avocado & 5.69 \\
Chicken egg yolk & 19.45 \\
Roasted peanuts & 4.86 \\
Goat fat & 70 \\
\hline${ }^{\mathrm{a}}$ Formula is obtained from the commercial rodent chow label. ${ }^{\mathrm{b}}$ Formula is \\
prepared based on ketogenic diet for rats, with the ratio of $8.6: 1$ portion of \\
fat:(carbohydrate + protein) [16].
\end{tabular}

2.2. Experimental Protocols. Male Wistar rats weighing 200-330 g age 3-4 months ( $n=15)$ were acclimated for 7 days in the laboratory before starting the experiment. At this stage, all rats received standard pellets and water ad libitum. Rats were cared for according to the standard for laboratory animal care, and all animal protocols have been approved by the Animal Ethics Committee of the Faculty of Medicine, Universitas Hasanuddin. Rats were divided into two groups. The first group $(n=8)$ received a standard diet, while the second group received the ketogenic diet for 60 days. This 60-day period of adult rat life is equivalent to $\sim 4$ years of human life [17]. Each rat was caged individually and offered $30 \mathrm{~g}$ of food per day ad libitum and not subjected to calorie restriction. The remaining food was weighed every morning to record the calorie intake of each rat. The blood samples were withdrawn following 60 days of treatments and prepared for further analysis.

2.3. Analysis of Blood Gas, Hematological Parameters, Organ Biomarkers, and Superoxide Dismutase Level. The blood gas analysis was performed on rat whole blood immediately following blood sampling with the use of the i-Stat ${ }^{\circledR}$ analyzer (Abbott ${ }^{\circledR}$ ). For hematological analysis, blood samples were collected using a $\mathrm{BD}^{\circledR}$ vacutainer with EDTA, centrifuged for $20 \mathrm{~min}$ with the rate of $3000 \mathrm{rpm}$ before analyzed using a hematology analyzer (Thermo Scientific ${ }^{\circledR}$ ). The organ biomarkers, such as alanine aminotransferase (ALT), aspartate aminotransferase (AST), creatinine, and urea were measured using Humalyzer 3500 (Human Global Diagnostic ${ }^{\circledR}$ ) according to the instruction on the reagent kits (Human $\left.{ }^{\circledR}\right)$. To measure plasma superoxide dismutase (SOD) level, the plasma was prepared based on instruction in Rat SOD for ELISA kit (Abbexa ${ }^{\circledR}$ ) and analyzed with the enzyme-linked immunosorbent assay (ELISA) reader (Thermo Scientific ${ }^{\circledR}$ ).

2.4. Analysis of Lipid Peroxidation Activity in Liver and Renal Tissues. At the end of the experiment, rats were anesthetized, euthanized, and laparotomy was performed. The 
liver and the kidneys of the rats were removed and immediately immersed in liquid nitrogen. Organs were weighed $400 \mathrm{mg}$ and homogenized before adding $2 \mathrm{~mL}$ of phosphate buffer solution $\mathrm{pH}$ 7.4. The mixture is centrifuged at $3000 \mathrm{rpm}$ for 20 minutes. The supernatant $(0.5 \mathrm{~mL})$ was mixed with $1 \mathrm{~mL}$ of $1 \%$ thiobarbituric acid and $1 \mathrm{~mL}$ of $1 \%$ trichloroacetic acid and heated to $100^{\circ} \mathrm{C}$ for 20 minutes. The mixture was then centrifuged at $3000 \mathrm{rpm}$ for 10 minutes to separate the residue. Organ lipid peroxidation was measured as malondialdehyde (MDA) level $(\lambda=530 \mathrm{~nm})$ using a UVVIS spectrophotometer (Agilent $\left.{ }^{\circledR}\right)$.

2.5. Statistical Analysis. The data obtained were analyzed using the SPSS IBM 23 software. Data distribution was examined using Kolmogorov-Smirnov to determine whether the data were normally distributed or not. The data that were normally distributed were subsequently analyzed using an independent $t$-test, while data that were not normally distributed were analyzed using the Mann-Whitney $U$ test. A significant difference was achieved if $p<0.05$ or very significant difference if $p<0.01$. All data were presented in mean \pm SEM.

\section{Results}

3.1. Long-Term Ketogenic Diet on Rats Causes Significant Weight Loss, Reduced Blood Glucose, and Increased Blood Ketone Levels. The food composition of the ketogenic pellet has far less carbohydrate (5.66\% vs $52.20 \%$ ) and much higher fat content $(86.19 \%$ vs $7.00 \%)$ compared to the standard diet (Table 2). The calorie of the standard chow is $5.85 \mathrm{kCal} / \mathrm{g}$, while that of the ketogenic pellet is $8.29 \mathrm{kCal} / \mathrm{g}$. The average of daily calorie intake per rat in each week is depicted in Table 3. It is found that the standard group consumed more amount of food than the ketogenic group; hence, the calorie intakes of both groups are quite similar despite the difference in calories per gram food.

The difference in the diet composition was found to significantly affect the body weight, blood glucose, and blood ketone levels in the male rats after 60-day intake. Table 4 shows the impact of ketogenic diet on rat body weight after 60 days. While all rats fed with standard diet gained weight after 2 months (on average $25 \%$ increase from baseline weight), the ketogenic-fed rats experienced a weight loss by around $100 \mathrm{~g}$ from their baseline body weight ( $40 \%$ loss).

Apart from weight loss, the blood glucose level of ketogenic-fed rats was significantly lower compared to the standard diet group (Figure 1). At this stage, the value of blood glucose was $57 \pm 5.69 \mathrm{mg} / \mathrm{dl}$, suggesting a hypoglycemic condition of the ketogenic diet group. Meanwhile, the level of blood ketone markedly elevated in the ketogenic group, about 8 times higher than the standard rats (7.97 \pm 0.15 vs $0.34 \pm 0.02 \mathrm{mmol} / \mathrm{L})$.

3.2. Long-Term Ketogenic Diet Significantly Lowered Blood pH and Reduced Base Excess Level. The analysis of blood gas values demonstrates that the administration of the ketogenic diet for 60 days causes a significant alteration in blood gas
TABLE 2: The comparison of carbohydrate, fat, and protein contents of standard and ketogenic diets obtained from food analysis.

\begin{tabular}{lccc}
\hline Type of diet & Carbohydrate (\%) & Fat (\%) & Protein (\%) \\
\hline Standard & 52.20 & 7.00 & 15.25 \\
Ketogenic & 5.66 & 86.19 & 8.15 \\
\hline
\end{tabular}

homeostasis (Table 5). It was found there was a very significant decrease in blood $\mathrm{pH}$ of rats following 2 months of having a ketogenic diet compared to those fed with a standard diet $(p<0.01)$. The decrease in blood $\mathrm{pH}$ was not accompanied by significant changes in carbon dioxide pressure $\left(\mathrm{pCO}_{2}\right)$, oxygen pressure $\left(\mathrm{pO}_{2}\right)$, total carbon dioxide $\left(\mathrm{TCO}_{2}\right)$, and hemoglobin oxygen saturation $\left(\mathrm{SO}_{2}\right)$. Although the blood bicarbonate $\left(\mathrm{HCO}_{3}^{-}\right)$level of the ketogenic group insignificantly decreased $(19.74 \pm 2.54$ vs $22.75 \pm 0.79 \mathrm{mmol} / \mathrm{L}$ ), it was found that the group's base excess level was significantly lower compared to the standard group $(p<0.05)$.

\subsection{Long-Term Ketogenic Diet Induces Anemia in Male Rats.} The result of hematological analysis after receiving standard and ketogenic diets for 60 days is presented in Table 6. The ketogenic group appears to have slightly lower red blood cell (RBC) counts, significantly lower hemoglobin, and hematocrit, as well as significantly smaller mean corpuscular volume $(\mathrm{MCV})$ and mean corpuscular hemoglobin $(\mathrm{MCH})$ indices. These hematological abnormalities indicate that rats fed with the ketogenic diet were anemic.

\subsection{Long-Term Ketogenic Diet Does Not Significantly Alter the} Functions of Liver and Kidney. This study also measured the effect of a long-term ketogenic diet in rats on liver and renal functions. The result is presented in Figure 2. From the data, it is revealed that the levels of liver biomarkers, the alanine aminotransferase (ALT) and aspartate aminotransferase (AST), were not significantly different between the standard and ketogenic groups. However, when comparing the renal function test, a slight increase in plasma creatinine and urea levels was found in the ketogenic group compared to standard, although the difference was not statistically significant.

\subsection{Long-Term Ketogenic Diet Increases Lipid Peroxidation} and Reduces the Antioxidant Level. The level of lipid peroxidation and antioxidant activity could be a good indicator to reveal oxidative stress level in the system. In this study, it was found that the ketogenic diet in rats for 60 days may induce an increase in malondialdehyde (MDA) level in the liver and kidney (Figure 3). The increase of MDA level in both vital organs was very significant in the ketogenic group compared to standard $(p<0.01)$. The increase of MDA level in the ketogenic group was accompanied by a reduced level of antioxidant superoxide dismutase (SOD), which was $\sim 80 \%$ lower compared to that of standard $(p<0.01)$. 
TABLE 3: The average of daily calorie intake per rat each week in standard and ketogenic diet groups.

\begin{tabular}{|c|c|c|c|c|c|c|c|c|}
\hline \multirow{2}{*}{ Diet } & \multicolumn{8}{|c|}{ The calorie intake (kCal/day) } \\
\hline & Week I & Week II & Week III & Week IV & Week V & Week VI & Week VII & Week VIII \\
\hline Standard & 83.11 & 81.24 & 81.13 & 82.83 & 80.62 & 78.29 & 76.19 & 75.57 \\
\hline Ketogenic & 88.77 & 84.78 & 82.86 & 83.45 & 86.70 & 84.78 & 88.77 & 98.24 \\
\hline
\end{tabular}

TABLE 4: Changes in rat body weight after receiving standard and ketogenic diets for 60 days.

\begin{tabular}{lcccc}
\hline Type of diet & $N$ & Body weight & Mean \pm SEM $(\mathrm{g})$ & $p$ value \\
\hline \multirow{2}{*}{ Standard } & \multirow{2}{*}{8} & Baseline & $252 \pm 20.61$ & $319 \pm 19.35$ \\
\hline \multirow{2}{*}{ Ketogenic } & \multirow{2}{*}{7} & Posttreatment & $0.001^{*}$ \\
& & Baseline & $260 \pm 12.60$ & $157 \pm 06.40$ \\
\hline
\end{tabular}

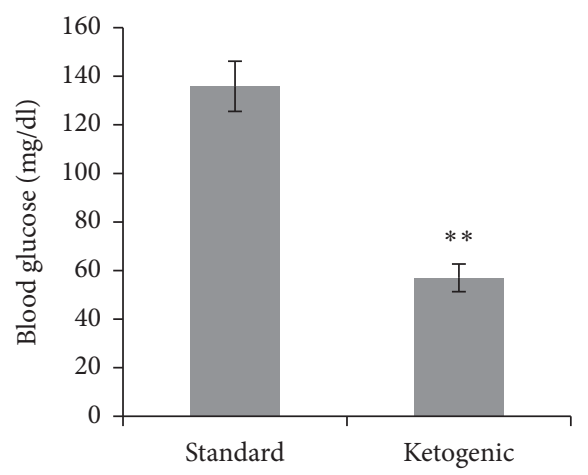

(a)

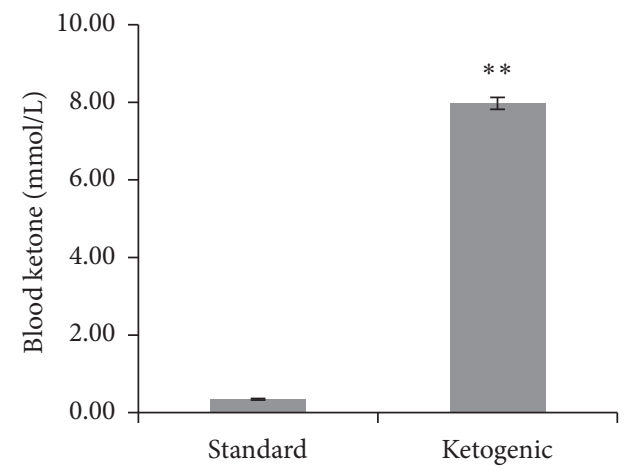

(b)

Figure 1: The level of blood glucose and blood ketone of rats consuming standard and ketogenic diet for 60 days. The symbol $* *$ implies a very significant difference $(p<0.01)$ between groups.

TABLE 5: The comparison of blood gas profiles of rats receiving standard and ketogenic diets for 60 days.

\begin{tabular}{lcccc}
\hline Blood gas & $N$ & Diet & Mean \pm SEM & $p$ value \\
\hline \multirow{2}{*}{$\mathrm{pH}$} & 8 & Standard & $7.52 \pm 0.01$ & \multirow{2}{*}{$0.001^{*}$} \\
\hline $\mathrm{pCO}_{2}(\mathrm{mmHg})$ & 7 & Ketogenic & $7.36 \pm 0.02$ & \\
\hline $\mathrm{pO}_{2}(\mathrm{mmHg})$ & 8 & Standard & $27.63 \pm 1.34$ & \multirow{2}{*}{1.00} \\
\hline $\mathrm{HCO}_{3}{ }^{-}(\mathrm{mmol} / \mathrm{l})$ & 7 & Ketogenic & $35.72 \pm 5.96$ & \\
\hline \multirow{2}{*}{$\mathrm{Base} \mathrm{excess}(\mathrm{mmol} / \mathrm{l})^{2}$} & 7 & Standard & $107.75 \pm 2.93$ & \multirow{2}{*}{0.32} \\
\hline \multirow{2}{*}{$\mathrm{TCO}_{2}(\mathrm{mmol} / \mathrm{l})$} & 7 & Ketogenic & $88.14 \pm 12.14$ & \\
\hline \multirow{2}{*}{$\mathrm{SO}_{2}(\%)$} & 8 & Ketandard & $22.75 \pm 0.79$ & \multirow{2}{*}{0.48} \\
\hline
\end{tabular}

\section{Discussion}

The ketogenic diet has gained public attention since it is first introduced as an alternative therapy for pharmacoresistant epilepsy [18]. Nowadays, the use of ketogenic diet has expanded beyond epileptic therapy; indeed, its use in healthy individuals has become more popular, especially to those
TABLE 6: The comparison of hematology profiles of rats receiving standard and ketogenic diets for 60 days.

\begin{tabular}{lcccc}
\hline Hematology parameters & $N$ & Diet & Mean \pm SEM & $p$ value \\
\hline \multirow{2}{*}{ RBC $\left(10^{6} / \mu \mathrm{L}\right)$} & 8 & Standard & $8.04 \pm 0.24$ & \multirow{2}{*}{0.33} \\
\hline \multirow{2}{*}{ Hemoglobin $(\mathrm{g} / \mathrm{dl})$} & 7 & Ketogenic & $7.65 \pm 0.29$ & \\
\hline \multirow{2}{*}{ Hematocrit $(\%)$} & 8 & Standard & $13.76 \pm 0.33$ & \multirow{2}{*}{$0.02^{*}$} \\
& 7 & Ketogenic & $11.98 \pm 0.54$ & \\
\hline \multirow{2}{*}{$\mathrm{MCV}(\mathrm{fL})$} & 8 & Standard & $39.90 \pm 0.97$ & \multirow{2}{*}{$0.001^{*}$} \\
\hline \multirow{2}{*}{$\mathrm{MCH}(\mathrm{pg})$} & 8 & Ketogenic & $32.77 \pm 1.69$ & \\
& 7 & Standard & $49.78 \pm 1.40$ & \multirow{2}{*}{$0.001^{* *}$} \\
\hline
\end{tabular}

who wish to lose weight. Unfortunately, the benefits of ketogenic diet may come with side effects. This study examined the long-term effects of ketogenic diet in a healthy male rat model to obtain more information about the potential complications of this type of diet.

Standard food with its high carbohydrate content allows the body to use glucose as the main source of energy. When carbohydrate intake is more than sufficient to meet the needs of ATP, the body will physiologically convert glucose into glycogen as energy stores in tissues. Consumption of a diet rich in carbohydrates will also cause an increase in the 


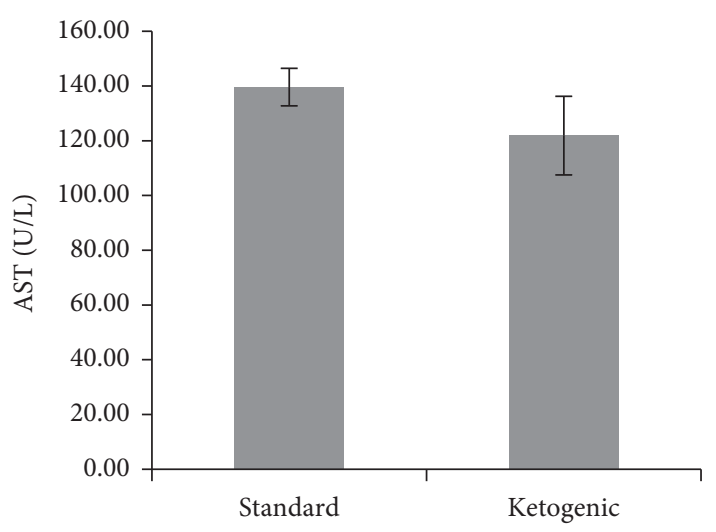

(a)

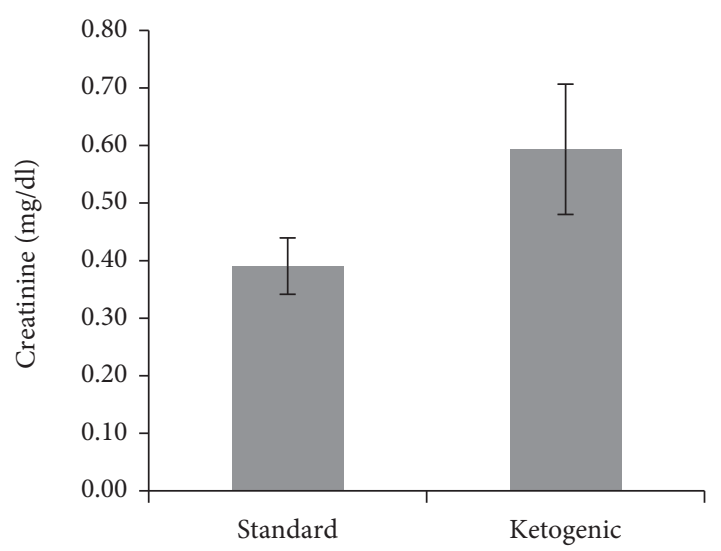

(c)

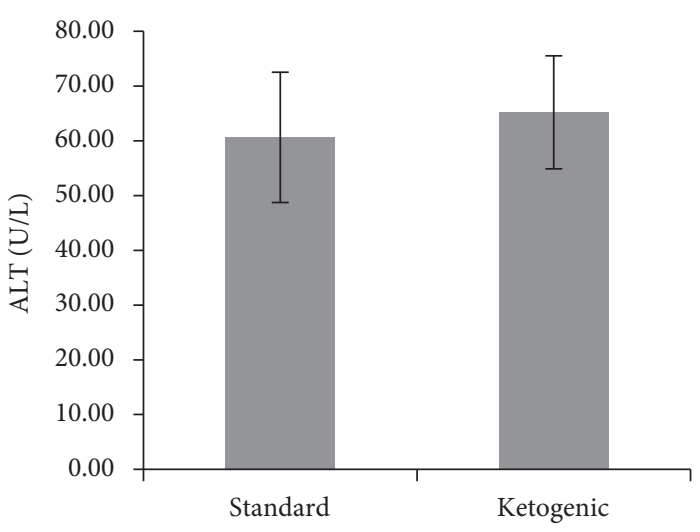

(b)

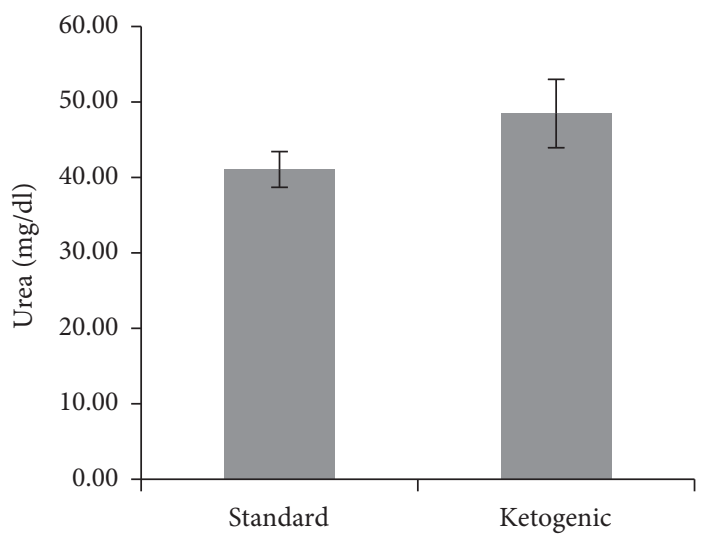

(d)

Figure 2: The level of aspartate aminotransferase (a), alanine aminotransferase (b), plasma creatinine (c), and plasma urea (d) in rats consuming standard and ketogenic diet for 60 days.

amount of fat deposited in adipose tissue under the skin or in the abdominal cavity. This is the main reason for the increase in body weight of rats fed with a standard diet.

On the other hand, rats treated with the ketogenic diet had a significant weight loss as a result of induced ketosis. Ketogenic diet with high fat, low protein, and low carbohydrate composition renders the body depends on the process of gluconeogenesis, the formation of noncarbohydrate glucose, to produce energy [19]. When the fatty acids (fat content) are mainly used to produce energy, it will induce the formation of ketone bodies, such as acetoacetate, beta-hydroxybutyrate, and acetone. The presence of ketosis in the ketogenic group was confirmed by a significantly higher level of blood ketone $(\sim 8 \mathrm{mmol} / \mathrm{L})$ and a significantly low blood sugar level $(<60 \mathrm{mg} / \mathrm{dl})$. Apart from weight loss, the rats also experience a decrease in $\mathrm{pH}$ or acidosis, which occurs as a result of increased blood ketone level [11]. The ketone bodies are acidic; thus, an increase in ketone bodies in circulation may induce acidosis $[11,20]$.

Anemia is not uncommon side effects of high-fat diets. This study also found reduced hematological indices, such as $\mathrm{RBC}$, hemoglobin, hematocrit, MCV, and $\mathrm{MCH}$ in rats fed with ketogenic diet for 60 days. Studies on epileptic children have revealed that ketogenic diet is more likely to cause anemia, which may occur due to dietary restriction, leading to copper deficiency $[21,22]$. However, this complication of the ketogenic diet can be managed with copper supplementation.

In this study, the administration of ketogenic diet in rats for 60 days did not significantly alter liver and kidney function. Nevertheless, the plasma creatinine and urea of the ketogenic-fed rats were somewhat higher than standard-fed rats, which may suggest a minor effect of the ketogenic diet on renal function. This effect could be more striking if the duration of the ketogenic diet administration is prolonged.

It is interesting that although the liver and renal function were not significantly altered, the lipid peroxidation activity in both organs significantly increased. This was indicated by a significantly higher MDA level of liver and renal tissues in ketogenic-fed rats compared to those with a standard diet. Increased activity of lipid peroxidation could be triggered by the elevation of reactive oxygen species (ROS) in the organs and incapability of the antioxidant enzyme activity to protect cell membranes from ROS-induced damage. This result could emerge as a potential threat to both organs should the diet be sustained longer than the period investigated. In line with this, the plasma concentration of superoxide dismutase (SOD) significantly reduced in ketogenic-fed animals $(p<0.01)$, suggesting the presence of oxidative stress induced by long-term ketogenic diet in rats. 


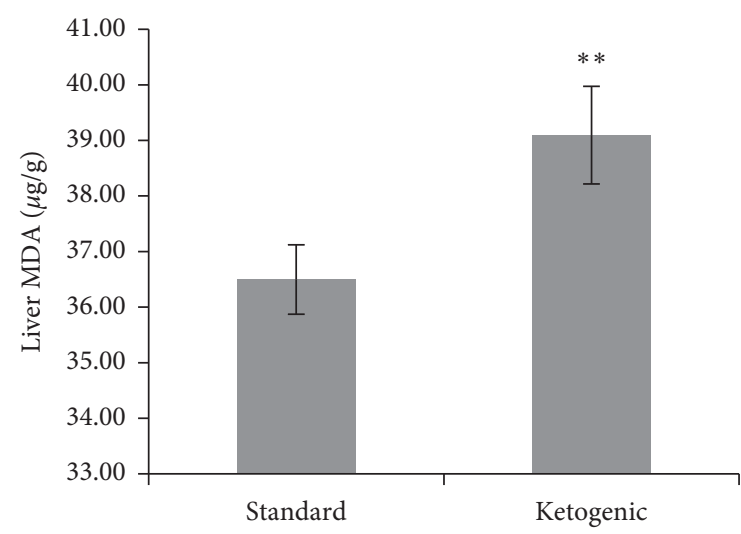

(a)

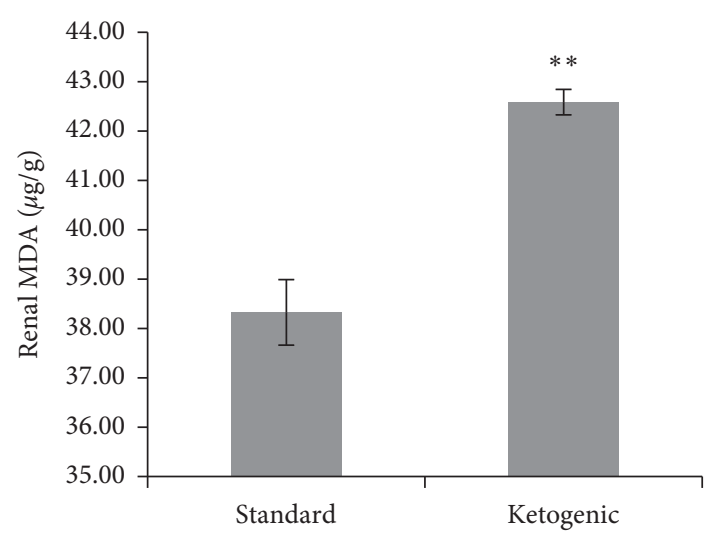

(b)

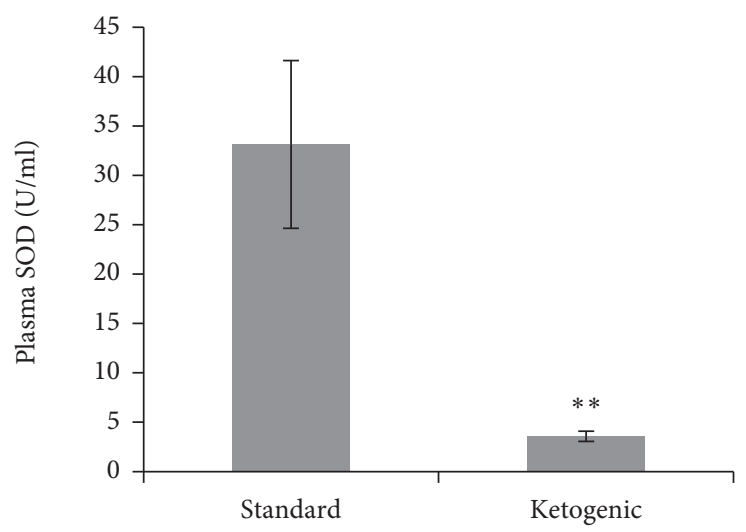

(c)

Figure 3: The level of liver malondialdehyde (a), renal malondialdehyde (b), and plasma superoxide dismutase (c) in standard and ketogenic-fed rats. The symbol $* *$ implies a very significant difference $(p<0.01)$ between groups.

The reason why a ketogenic diet may induce oxidative stress has been explained in several studies. Ketone bodies are known to stimulate the mitochondria to produce more ATP compared to glucose [23-25]. However, fat metabolism requires more complex processes, such as reduction, oxidation, hydroxylation, and conjugation, which may elevate the production of reactive oxygen species (ROS) in the liver cells $[26,27]$. If the release of ROS is in balance with the body's antioxidant activities, the occurrence of oxidative stress can be prevented. In contrast, if ROS formation has exceeded antioxidant levels, the free radicals will attack macromolecules, such as, proteins, polysaccharides, DNA, and cell membranes that contain polyunsaturated fatty acids, leading to cellular damage [28]. This study shows an increase in liver and renal MDA levels, which is accompanied by a decrease in plasma SOD after 60-day consumption of ketogenic diet. This might implicate a precaution on the longterm use of the ketogenic diet.

\section{Conclusions}

Despite the weight loss, low blood sugar, and high blood ketone, sustainable consumption of keto diet for 60 days in rats also instigated some concerning effects such as metabolic acidosis, anemia, and decreasing plasma antioxidant enzyme level. It is interesting that albeit a significant increase in lipid peroxidation activity on the liver and kidney, both organ functions were remained intact, at least during the period investigated.

\section{Data Availability}

The data used to support the findings of this study are available from the corresponding author upon request.

\section{Conflicts of Interest}

The authors declare no conflicts of interest.

\section{Acknowledgments}

This publication was made possible by a block grant from the Faculty of Medicine, Hasanuddin University, Indonesia.

\section{References}

[1] C. Kosinski and F. Jornayvaz, "Effects of ketogenic diets on cardiovascular risk factors: evidence from animal and human studies," Nutrients, vol. 9, no. 5, p. 517, 2017.

[2] R. Alessandro, B. Gerardo, L. Alessandra et al., "Effects of twenty days of the ketogenic diet on metabolic and respiratory parameters in healthy subjects," Lung, vol. 193, no. 6, pp. 939-945, 2015. 
[3] J. M. Rho, "How does the ketogenic diet induce anti-seizure effects?" Neuroscience Letters, vol. 637, pp. 4-10, 2017.

[4] S.-C. Mak, C.-S. Chi, and C.-J. Wan, "Clinical experience of ketogenic diet on children with refractory epilepsy," Acta Paediatrica Taiwanica, vol. 40, pp. 97-100, 1999.

[5] H. M. Dashti, T. C. Mathew, M. Khadada et al., "Beneficial effects of ketogenic diet in obese diabetic subjects," Molecular and Cellular Biochemistry, vol. 302, no. 1-2, pp. 249-256, 2007.

[6] R. R. Nazarewicz, W. Ziolkowski, P. S. Vaccaro, and P. Ghafourifar, "Effect of short-term ketogenic diet on redox status of human blood," Rejuvenation Research, vol. 10, no. 4, pp. 435-440, 2007.

[7] K. J. Bough and J. M. Rho, "Anticonvulsant mechanisms of the ketogenic diet," Epilepsia, vol. 48, pp. 43-58, 2007.

[8] J. E. Hall, Guyton and Hall Textbook of Medical Physiology E-Book, Elsevier Health Sciences, Amsterdam, Netherlands, 2015.

[9] P. Puchalska and P. A. Crawford, "Multi-dimensional roles of ketone bodies in fuel metabolism, signaling, and therapeutics," Cell Metabolism, vol. 25, no. 2, pp. 262-284, 2017.

[10] R. T. Lagua and V. S. Claudio, Nutrition and Diet Therapy Reference Dictionary, Springer, Berlin, Germany, 2012.

[11] T. Fukao, G. D. Lopaschuk, and G. A. Mitchell, "Pathways and control of ketone body metabolism: on the fringe of lipid biochemistry," Prostaglandins, Leukotrienes and Essential Fatty Acids, vol. 70, no. 3, pp. 243-251, 2004.

[12] S. A. Noeman, H. E. Hamooda, and A. A. Baalash, "Biochemical study of oxidative stress markers in the liver, kidney and heart of high fat diet induced obesity in rats," Diabetology \& Metabolic Syndrome, vol. 3, p. 17, 2011.

[13] P. J. Raubenheimer, M. J. Nyirenda, and B. R. Walker, "A choline-deficient diet exacerbates fatty liver but attenuates insulin resistance and glucose intolerance in mice fed a highfat diet," Diabetes, vol. 55, no. 7, pp. 2015-2020, 2006

[14] G. Vial, H. Dubouchaud, K. Couturier et al., "Effects of a highfat diet on energy metabolism and ROS production in rat liver," Journal of Hepatology, vol. 54, no. 2, pp. 348-356, 2011.

[15] S. Kume, T. Uzu, S.-I. Araki et al., "Role of altered renal lipid metabolism in the development of renal injury induced by a high-fat diet," Journal of the American Society of Nephrology, vol. 18, no. 10, pp. 2715-2723, 2007.

[16] Q. Kashiwagi, C. E. Stafstrom, D. D. Fu, Y. Hu, and G. L. Holmes, "Detrimental effects of the ketogenic diet on cognitive function in rats," Pediatric Research, vol. 55, no. 3 , pp. 498-506, 2004.

[17] P. Sengupta, "The laboratory rat: relating its age with human's," International journal of preventive medicine, vol. 4, no. 6, p. 624, 2013.

[18] M. S. Duchowny, "Food for thought: the ketogenic diet and adverse effects in children," Epilepsy Currents, vol. 5, no. 4, pp. 152-154, 2005.

[19] K. D. Hall, K. Y. Chen, J. Guo et al., "Energy expenditure and body composition changes after an isocaloric ketogenic diet in overweight and obese men," The American Journal of Clinical Nutrition, vol. 104, no. 2, pp. 324-333, 2016.

[20] A. W. C. Ravussin, I. A. Walcutt, and J. W. Sander, "An acidosis-sparing ketogenic (ASK) diet to improve efficacy and reduce adverse effects in the treatment of refractory epilepsy," Epilepsy \& Behavior, vol. 74, pp. 15-21, 2017.

[21] H. Rashidian, Y. M. C. Liu, M. T. Geraghty, J. Kobayashi, E. J. Donner, and R. J. Klaassen, "Severe neutropenia and anemia in a child with epilepsy and copper deficiency on a ketogenic diet," Pediatric Neurology, vol. 76, pp. 93-94, 2017.
[22] A. Chin, "Copper deficiency anemia and neutropenia due to ketogenic diet," Pediatrics, vol. 141, Article ID e20173286, 2018.

[23] S. Ma, Q. Huang, K. Yada, C. Liu, and K. Suzuki, “An 8-week ketogenic low carbohydrate, high fat diet enhanced exhaustive exercise capacity in mice," Nutrients, vol. 10, no. 6, p. 673, 2018.

[24] A. M. Holland, W. C. Kephart, P. W. Mumford et al., "Effects of a ketogenic diet on adipose tissue, liver, and serum biomarkers in sedentary rats and rats that exercised via resisted voluntary wheel running," American Journal of PhysiologyRegulatory, Integrative and Comparative Physiology, vol. 311, no. 2, pp. R337-R351, 2016.

[25] K. Kavazis, H. Saito, K. Sumi, Y. Sakamoto, Y. Tachi, and K. Iida, "Short-term and long-term ketogenic diet therapy and the addition of exercise have differential impacts on metabolic gene expression in the mouse energy-consuming organs heart and skeletal muscle," Nutrition Research, vol. 60, pp. 77-86, 2018.

[26] L. Adriani, A. Rochana, A. Yulianti, A. Mushawwir, and N. Indrayani, "Profil serum glutamate oxaloacetat transaminase (SGOT) and glutamate pyruvate transaminase (SGPT) level of broiler that was given noni juice (Morinda citrifolia) and palm sugar (Arenga piata)," LucrariStiintifice-SeriaZootehnie, vol. 62, pp. 101-105, 2014.

[27] J. R. Garbow, J. M. Doherty, R. C. Schugar et al., "Hepatic steatosis, inflammation, and ER stress in mice maintained long term on a very low-carbohydrate ketogenic diet," American Journal of Physiology-Gastrointestinal and Liver Physiology, vol. 300, no. 6, pp. G956-G967, 2011.

[28] B. Poljsak, D. Šuput, and I. Milisav, "achieving the balance between ROS and antioxidants: when to use the synthetic antioxidants," Oxidative Medicine and Cellular Longevity, vol. 2013, Article ID 956792, 11 pages, 2013. 